\title{
Modal Analysis of the Numerical Control Machine Based on Grid Technology
}

\author{
Dongfang $\mathrm{Hu}^{1, \mathrm{a}^{*}}$, Yafei Zheng ${ }^{1, \mathrm{~b}}$ and Yan Zhao ${ }^{2, \mathrm{c}}$ \\ ${ }^{1}$ School of Mechatronics Engineering, Henan University of Science and Technology, Luoyang 471003, \\ China \\ ${ }^{2}$ LandGlass Technology Co., Ltd, Luoyang 471003, China \\ ahdf@haust.edu.cn, bilovfe@163.com, chlxc-2009@163.com
}

Keywords: Vibration; Modal Analysis; Column; Finite Element; Grid Technology

\begin{abstract}
Vibration is a major problem in the design of high precision machining machine tool, which has great influence on the machining precision. So it is very necessary to carry out the modal analysis of the column components. The three-dimensional finite element model of the column was established, and the modal analysis was carried out through ANSYS. And first five order natural frequency and vibration mode of the column were obtained, which is helpful for us to understand the dynamic performance of the column and to improve the machining accuracy of the machine. The complex device of the machine tool requires the corresponding higher computing power as the basis of the analysis. The application of the grid technology was created to solve the problem of computing power. A computer grid platform was set up and distributed parallel computing software of ANSYS was deployed on this platform. It proved that this platform make analysis rapid and accurate.
\end{abstract}

\section{Introduction}

The numerical control machine tools are developing for the high-speed, high-efficiency, high-precision, and high-integration. In many cases, its performance of vibration is an essential factor to influence the performance and improvement of the machine tool, especially in machine-tools with high-speed and high-precision. Therefore, how to limit the effect of vibration of machine or to improve its anti-vibration performance has become a key factor for success of design [1].

Grid technology was called the third-generation network technology. It is a new era of internet technology after the computer equipment communication and Web technology, which acquired communication under traditional Internet, to achieve the communication of wide area network environment. Computers, databases, software, digital equipment are taken as infrastructure to work together on grid technology. Thus, the grid is a kind of integrated resources and environment of services. The target of grid is to actualize a "virtual super computer machine", which integrates computers to work together at the same time in different locations on internet. The grid realizes comprehensive information sharing of computing resources, storage resources, data resources, software resources, communication resources, knowledge resources and expert resources and so on [2].

The column is an important component of the machine tool. When the natural frequency of the column unit is equal or close to the excitation frequency, the amplitude will sharp increase, and the resonance will be happened. Therefore, the natural frequencies of the column unit should not be ignored as an evaluation of the column unit. Since the machine column is a relatively complex mechanical device, the accuracy exceedingly depends on the computer models whether it is similar to its prototype, and the size of the cell division, which requires correspondingly higher computing power as the basis for analysis. Until now, when conducting machine-tools performance research, single machine analysis, which cannot solve the problem of computing power and it takes a long time, is used widely in our nation [3]. So it is necessary to attempt the grid technology to research the machine tool column. A computer grid platform should be set up to satisfy the analysis. As long as deploying 
ANSYS Linux distributed parallel computing software platform on this analysis, it could make analysis rapid and accurate.

The object of this study is a large strong horizontal milling machine in an enterprise. Since the process of milling belongs to accurate processing which requires high precision, column is an important part of machine tool. So dynamic performance of column has a great influence on processing precision. Therefore, dynamic analysis of column can help us understand its natural frequencies and mode shapes, which are conducive to avoid unnecessary losses caused by resonance. Modal analysis is the main method for research of dynamic performance of column. Its main content is to determine the vibration characteristics of the column that is the natural frequency and main vibration mode, whereby we can determine whether the modes could affect the machining accuracy. At last, we compete the optimizing design of machine tool column, so that it could meets the requirements of the enterprise.

\section{Theory of Modal Analysis}

$$
[\boldsymbol{M}]\{\ddot{\boldsymbol{X}}(t)\}+[\boldsymbol{C}]\{\dot{\boldsymbol{X}}(t)\}+[\boldsymbol{K}]\{\boldsymbol{X}(t)\}=\boldsymbol{f}(t)
$$

In the type: $\mathrm{M}, \mathrm{C}$ and $\mathrm{K}$ stand for the quality of systems, damping and rigid matrix, $\mathrm{x}(\mathrm{t})$ and $\mathrm{f}(\mathrm{t})$ stand for displacement response vector and the inspiration force vector.

When the $f(t)=0$, general solution of the homogeneous equation will reflect the free vibration characteristics of the system, and its corresponding characteristic equation system solution features will reflect the inherent characteristics of the structure [4].

When the $\mathrm{f}(\mathrm{t}) \neq 0$, it is non-homogeneous equation. Solutions of the characteristic equation will reflect the input load characteristics. Natural frequency and main vibration mode are a natural property of the vibration system. It is necessary to solve this problem by studying non-damped free vibration. [5]

$$
\begin{aligned}
& {[\boldsymbol{M}]\{\ddot{\boldsymbol{X}}(t)\}+[\boldsymbol{K}]\{\boldsymbol{X}(t)\}=0} \\
& \mathbf{X}=\boldsymbol{\Phi} \sin \omega\left(t-t_{0}\right)
\end{aligned}
$$

In the type: $\Phi$ is a vector of $\mathrm{N}$-order; $\omega$ is the vibration frequency of the vector; $t$ is the variable of time, $\mathrm{t}_{0}$ is time constant which is determined by the initial conditions.

$$
K \boldsymbol{\Phi}-\omega^{2} \boldsymbol{M \Phi}=0
$$

To solve the equations above, $\Phi$ and $\omega$ are gained, and solution of $\left(\omega_{1}{ }^{2}, 1\right),\left(\omega_{2}{ }^{2}, 2\right), \ldots,\left(\omega_{n}{ }^{2}, \Phi_{n}\right)$ is obtained. $\omega_{1}, \omega_{2}, \ldots, \omega_{n}$ represents the $\mathrm{N}$ inherent frequency of the system, and the feature vector 1 , $2, \ldots, \Phi_{\mathrm{n}}$ represents $\mathrm{N}$ inherent vibration of the system.

After the computer grid platform was built, ANSYS11.0 parallel computing software for Linux is deployed on the dawn of TC4000 cluster. Node 1 is set to the primary, and node 2 to node 8 are taken as sub-node during the installation of the cluster. When the cluster received the assignment, the master node will distribute the data of assignment to each child node, and then each node operates separately and gives back the results of the operation to the primary node [6].

\section{Model of Milling and Boring Machine Tools}

Finite element analysis is based on the finite element model. whereas finite element model is a geometric model which is generated by the element mesh. Therefore, geometric model should be established firstly and finite element model is obtained from the geometric model.

Materials of Overall Model. The smaller parts of the model should be simplified. The main material of the column is HT300, its material properties as follows:

Density: 7.8e 3 elastic modulus: 1.3e11Pa; Poisson ratio: 0.24; track coefficient of friction: 0.2. 
Establish of Overall Geometric Model. The establishment of overall geometric model is still very hard even in ANSYS. According to the principle of simplified model, the details of the column, which are little effect on overall geometric model, are simplified. Such as parts of the entity as follows, chamfers, curving of castings, and bolt holes on the bottom [7].

The simplified geometric model sees in Fig. 1, and finite element model in ANSYS sees in Fig. 2.

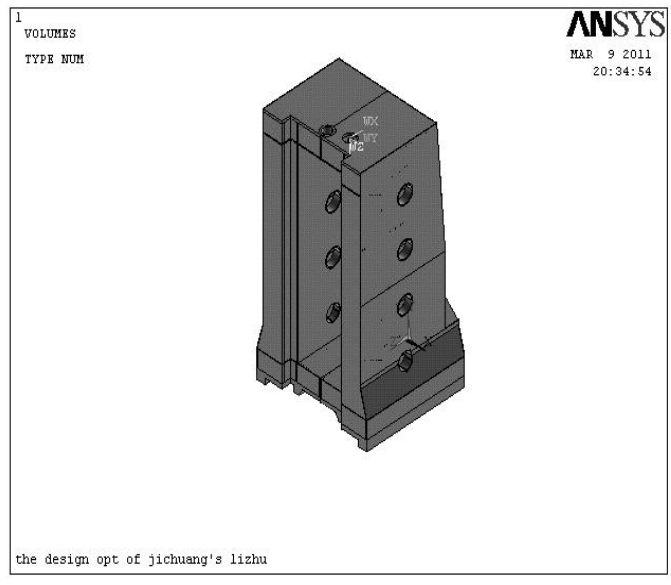

Figure 1. Simplified geometric model

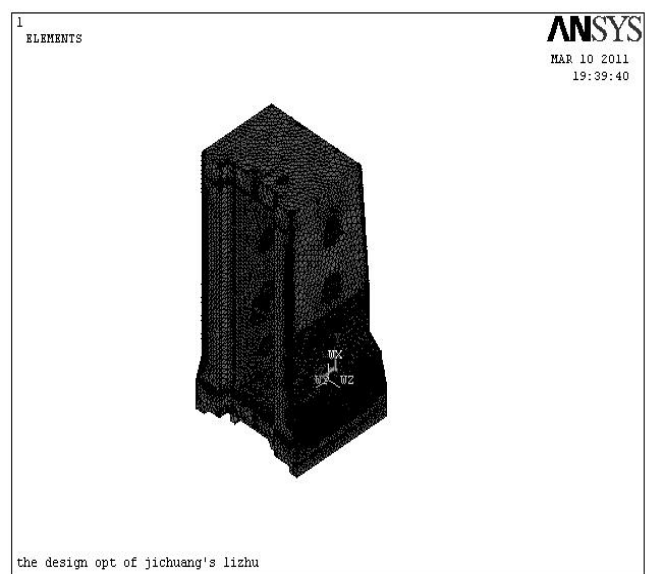

Figure 2. Finite element model

Establish of Milling and Boring Finite Element Model. For such a complex structure of the machine, it is difficult to obtain a finite element model by using maps or sweeping method if choose the hexahedral elements. It takes the following principles to obtain the finite element model.

The Choice of Tetrahedral Solid Element. According to the material of the column, HT300 is orthogonal isotropic material. 3D solid elements such as Solid45, Solid92, Solid95 and so on, are usually used [8]. As the accuracy of analysis is a great relationship with the number of unit nodes, we should try to choose higher-order unit. The character of common 3D elements sees table 1.

Table 1 The character of common 3D elements

\begin{tabular}{cccccc}
\hline Unit & abbreviation & node & $\begin{array}{c}\text { complete/curtail } \\
\text { integration }\end{array}$ & $\begin{array}{c}\text { Initial } \\
\text { stress }\end{array}$ & remark \\
\hline Solid45 & Entity element & 8 & $Y / Y$ & $Y$ & Orthogonal isotropic material \\
Solid92 & Tetrahedron entity element & 10 & $Y / Y$ & $Y$ & Orthogonal isotropic material \\
Solid95 & Entity unit & 20 & $Y / Y$ & $Y$ & Solid45 high order element \\
\hline
\end{tabular}

According to the structure of milling and boring machine, if the Solid45 unit was chosen, the accuracy is the lowest. The number of nodes depends on the type of unit chose, especially when the number of Solid95 unit nodes is 20, the number of nodes Solid92 unit nearly 0.5 times less than Solid95 unit. For milling and boring machine, the number of nodes is more and more, but the accuracy is not different between Solid95 and Solid92 units. Therefor the Solid95 is usually replaced by Solid92 during the calculations [9].

Units on Contact Surface. When contact surface is set through contacting guide, it will automatically generate the corresponding contact unit. Contact element is on the surface of the contact layer of the model unit cell.

There are two surfaces in contact surface. The two surfaces are respective the target surface and the contact surface. To avoid appear errors, symmetrical contact should be set, but the calculation process has been increased by nearly one fold. The contact surface is with Contact174, and the target surface is with Target170. The character of common contact \& target elements sees Table 2. 
Table 2 The character of common contact \& target elements

\begin{tabular}{ccccc}
\hline Unit name & Abbreviation & Node & Node DOF & Remark \\
\hline Target170 & 3Dtarget element & 8 & UTVMR & solid elements which can simulate complex shapes \\
Contact174 & $\begin{array}{c}\text { 3D8contactsurface } \\
\text { element }\end{array}$ & 8 & UTVMR & Coulomb friction and shear stress friction. \\
\hline
\end{tabular}

\section{Sets of Analysis Options}

As the analysis of milling and boring is kinetic analysis, thus Antype is set to Modal. The column is connected by screws with the bottom surface of the machine tool. It is regarded as the part of machine tool. It is similar to a cantilever structure in kinetics. The six degrees of freedom of the column are limited at the bottom. Therefore, according to the methods of surface constraints, UY, UZ, UX, ROTX, RTOY, RTOZ direction of constraints are added. After constraints are added, the bottom surface is unable to move against the machine bed. Under such circumstances, the obtained results of analysis are the vibration of column against machine bed.

\section{Resolver Selection}

Subspace method: with high accuracy but slow calculation speed, it is applied to solve large symmetric eigenvalue problems.

Block Lanczos: with high accuracy but calculation speed, its scope is as the same as subspace.

Deduced: with high calculation speed but low accuracy, the accuracy and speed of calculation depends on the number and position of the selected master degrees of freedom.

Un-symmetric: it is suitable for problems of the rigid and mass matrices un-symmetric. Some of the high frequency modes may be lost through this method.

Damping: It is applied to the problem of damping that cannot be ignored [10].

QR Damping: it is suitable for solving large problems of damping system. Its accuracy of calculating depends on the number of modes extracted.

As to the Block Lanczos method's character of high calculation speed and accuracy, it is usually used to solve problems of large structure. Therefor BlockLanczos is applied to establish the finite element model to solve the milling mode in ANSYS.

\section{Vibration Mode of the Column}

The vibration of the column can be expressed as a linear combination of the natural mode of each order. The lower order natural vibration mode is higher than the high order to the vibration of the column. The low order mode plays a decisive role in the dynamic characteristics of the column. So the analysis and calculation of the vibration characteristics of the column is usually taken in the first 1-10 order. Top 10-order modal frequency of column sees table 3.

Table 3 Top 10-order modal frequency of column

\begin{tabular}{ccc}
\hline Sub step response & Frequency & Load step \\
\hline 1 & 6.9820 & 1 \\
2 & 10.920 & 1 \\
3 & 14.832 & 1 \\
4 & 59.118 & 1 \\
5 & 89.713 & 1 \\
6 & 102.52 & 1 \\
7 & 140.87 & 1 \\
8 & 167.57 & 1 \\
9 & 217.24 & 1 \\
10 & 268.38 & 1 \\
\hline
\end{tabular}




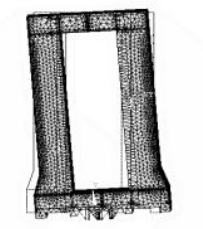

First-order mode

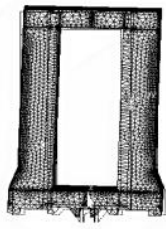

Second-order mode

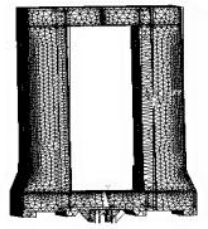

Third-order mode

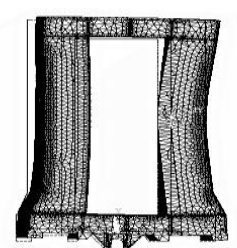

Fourth-order mode

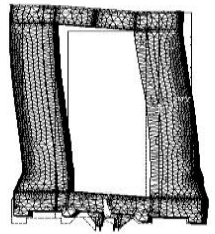

Fifth-order mode

Figure 3. N-order mode

\section{Conclusion}

As what can be seen from the mode of vibration, the deformation of upper part of the column is relatively obvious. The deformation gradually decreases from the top to the down. The maximum displacement of deformation is mostly in the area of the top of the column. Due to the suspension spindle box, the column is forced a larger bending moment. So it is necessary to strengthen the stiffness of the column by adding the reinforcement to reduce vibration and deformation. Considering to set aside a considerable space to install the counterweight, the inside reinforced rib is chosen, which may ensure the strength and rigidity of the machine tool.

Taking full advantage of ANSYS to analyze the mode of the column of the milling machine, it proved that the method is simple and convenient, and the calculation is fast. The obtained model is intuitive and easy to understand.

\section{Acknowledgements}

The authors gratefully acknowledge the National Nature Science Foundation (Project No. 51475142), the Key Technology R \& D Program of Henan Province (Project No. 13A520232), and the Major and Previous Pre-research Project of Henan University of Science and Technology (Project No. 2011CX016).

\section{References}

[1] Y.L. Zhang, X.D. WANG, G.Q. CAI, et al: Modular Machine Tool \& Automatic Manufacturing Technique, 2004, No.12, p.18 (In Chinese).

[2] Z. Qiao: Research on Resource Discovery in Grid Environment (MS. Lanzhou University of Technology, China 2009), p.1.

[3] Y.J. Wang: Research and application for NC machine's performance analysis based on the computer grid technology (MS. Henan University of Science and Technology, China 2009), p.1.

[4] X.H. Geng, Y.T. Liu, and S.J. Wang: Equipment Manufacturing Technology, 2010, No.01, p.71 (In Chinese).

[5] Y. Hou, G.H. Zeng, and G.F. Li: Journal of Hubei University of Technology, 2010, No.04, p.118 (In Chinese).

[6] ANSYS.INC: Distributed ANSYS Guide (Help Files of ANSYS, America 2006).

[7] X.Y. Wang, T. Ning, and K. Wang: Mechanical Engineer, 2009, No.02, p.83 (In Chinese).

[8] Y.X. Zhang: Research of stress analysis to the hydraulie support (MS. Xi'an University of Science and Technology, China 2011), p.45.

[9] Y.J. Wang, J.D. Shang, and D.F. Hu: Mechanical Engineer, 2009, No.10, p.63 (In Chinese).

[10]G.J. Zhang: Dynamic and Anti-seismic Analysis of Liquid Storage Tank (MS. Beijing University of Chemical Technology, China 2010), p.35. 